\title{
Supersolid edge and bulk phases of a dipolar quantum gas in a box
}

\author{
S. M. Roccuzzo $\odot$, S. Stringari, and A. Recati* \\ INO-CNR BEC Center and Dipartimento di Fisica, Università degli Studi di Trento, 38123 Povo, Italy \\ and Trento Institute for Fundamental Physics and Applications, INFN, 38123, Trento, Italy
}

(Received 2 April 2021; revised 29 June 2021; accepted 3 November 2021; published 3 February 2022)

\begin{abstract}
We investigate the novel density distributions acquired by a dipolar Bose-Einstein condensed gas confined in a box potential, with special focus on the effects of supersolidity. Different from the case of harmonic trapping, the ground-state density reveals a strong depletion in the bulk region and an accumulation of atoms near the walls, well separated from the bulk, as a consequence of the competition between the attractive and the repulsive nature of the dipolar force. In a quasi-two-dimensional geometry characterized by cylindrical box trapping, we observe the emergence of a ringlike configuration near the boundary of the box, revealing peculiar supersolid and crystal effects in a useful range of parameters. In the case of square box trapping, the density oscillations along the edges, caused by the enhanced accumulation of atoms near the vertices, exhibit interesting analogies with the case of box-trapped quasi-one-dimensional configurations. For sufficiently large values of the atom number, the bulk region can also exhibit supersolidity, the resulting geometry reflecting the symmetry of the confining potential even for large systems.
\end{abstract}

DOI: 10.1103/PhysRevResearch.4.013086

\section{INTRODUCTION}

Bose-Einstein-condensed atomic gases have proved to be an invaluable tool for the study of the physics of manybody systems. However, while typical many-body problems consider translationally invariant systems in the thermodynamic limit, Bose-Einstein condensates (BECs) are ordinarily realized in small, inhomogeneous samples confined by harmonic potentials [1]. Although harmonic trapping allows the study of relevant properties of these many-body systems (e.g., collective excitations [2,3], superfluid properties [4-6], and quantized vortices [7-9]), other important properties, like sound propagation or critical behaviors, can be better studied in uniform systems. For these reasons, Bose-Einstein condensation in "box" potentials has been an emerging topic of research in recent years, leading to the realization of uniform BECs in gases of alkali atoms and first important measurements in both three-dimensional and two-dimensional configurations [10-17].

The achievement of BECs of magnetic atoms in harmonic traps [18-21] opened the way to the study of the very peculiar physics of dipolar BECs, which includes a geometry dependence of phase diagram stability [22], a rotonized excitation spectrum [23-26], quantum droplets [27-30], and, more recently, supersolidity [31-36]. Supersolidity in dilute atomic gases was first experimentally achieved in BEC gases confined in optical resonators [37] and in spin-orbit-coupled gases [38]. While most of the theories for supersolidity are

\footnotetext{
*Corresponding author: alessio.recati@ino.it
}

Published by the American Physical Society under the terms of the Creative Commons Attribution 4.0 International license. Further distribution of this work must maintain attribution to the author(s) and the published article's title, journal citation, and DOI. developed for infinite systems, all the experiments have been so far conducted in trapped systems.

The natural question that arises is, therefore, how a dipolar gas behaves in a box potential and to what extent its configurations mimic their thermodynamic counterparts. First theoretical investigations carried out in the deep superfluid phase [39] have pointed out the peculiar phenomenon of accumulation of the density distribution near the boundary as a consequence of the repulsive behavior of the aligned dipoles. This effect is strongly reduced in the presence of transverse harmonic trapping because of the high energetic cost for dipoles to move away from the center of the trap. The present work is based on a proper generalization of the Gross-Pitaevskii equation (GPE) which contains the terms due to the Lee-Huang-Yang (LHY) correction to the mean-field equation of state, and it mainly focuses on the new supersolid features exhibited by the system in the presence of the box. We find that in quasi-two-dimensional geometries the accumulation along the border is enhanced in the regimes where the LHY correction is relevant, creating edges pretty well separated from the bulk. For a relatively small number of atoms, the bulk remains in a low-density superfluid phase, while the edges can show typical supersolid or droplet crystal structures (see Fig. 1). Increasing the atom density leads to a supersolid bulk region, while the edges can be found to be in a high-density superfluid phase (see Fig. 5). Moreover, the lattice emerging in the bulk has not been in general a triangular (or honeycomb) pattern, as expected for an infinite system [40], but its structure is dictated by the shape of the confining box potential even for relatively large systems.

\section{THE MODEL}

We start our exploration by considering the case of a quasi-two-dimensional dipolar BEC, obtained by imposing 


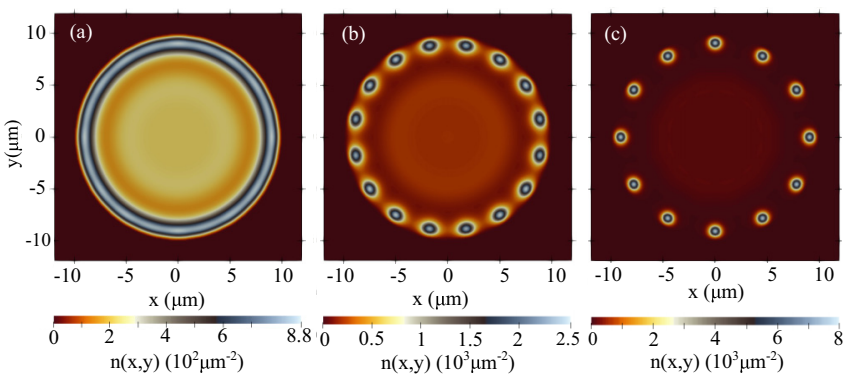

FIG. 1. Ground-state integrated density profiles $n(x, y)=$ $\int d z|\Psi(x, y, z)|^{2}$ for a gas of $10^{5}$ atoms of ${ }^{164}$ Dy confined in the polarization direction by a harmonic potential of frequency $\omega_{z}=(2 \pi) 100 \mathrm{~Hz}$, and by a box potential in the $x-y$ plane, with the shape of a circle of radius $\mathrm{R}=10.185 \mu \mathrm{m}$. The value of $\epsilon_{d d}$ for panels $(\mathrm{a}-\mathrm{c})$ is, respectively, 1.32, 1.404, and 1.467. The height of the box is fixed to $V_{0}=100 \hbar \omega_{z}$

a harmonic confinement only in the polarization direction ( $z$ axis). At zero temperature, the dipolar BEC is described by a macroscopic wave function $\Psi(\mathbf{r}, t)$, whose square modulus gives the local density of the system and which obeys to the so-called extended Gross-Pitaevskii equation (eGPE) [41,42],

$$
\begin{aligned}
& i \hbar \frac{\partial \Psi(\mathbf{r}, t)}{\partial t}=\left[-\frac{\hbar^{2} \nabla^{2}}{2 m}+V_{\mathrm{ext}}(\mathbf{r})+g|\Psi(\mathbf{r}, t)|^{2}\right. \\
& \left.+\int d \mathbf{r}^{\prime} V_{d d}\left(\mathbf{r}-\mathbf{r}^{\prime}\right)\left|\Psi\left(\mathbf{r}^{\prime}, t\right)\right|^{2}+\gamma\left(\epsilon_{d d}\right)|\Psi(\mathbf{r}, t)|^{3}\right] \Psi(\mathbf{r}, t),
\end{aligned}
$$

where $V_{\text {ext }}(\mathbf{r})$ is the trapping potential, $g=4 \pi \hbar^{2} a / m$ is the coupling constant fixed by the s-wave scattering length $a$, and $V_{d d}(\mathbf{r})=\frac{\mu_{0} \mu^{2}}{4 \pi} \frac{1-3 \cos ^{2} \theta}{|\mathbf{r}|^{3}}$ is the dipole-dipole interaction between two identical magnetic dipoles $\mu$ aligned along the $z$ axis ( $\theta$ is the angle between $\mathbf{r}$ and the $z$ axis). A key dimensionless parameter is $\epsilon_{d d}$, defined as

$$
\epsilon_{d d}=\frac{\mu_{0} \mu^{2}}{3 g},
$$

which measures the relative strength of the dipolar and the contact interaction. Experimentally, the parameter $\epsilon_{d d}$ is tuned by changing the value of the $s$-wave scattering length $a$, thanks to the occurrence of a Feshbach resonance. The last term in the eGPE equation is the local-density approximation of the LHY correction to the ground-state energy of the system [41,42], with

$$
\gamma\left(\epsilon_{d d}\right)=\frac{16 g a^{\frac{3}{2}}}{3 \sqrt{\pi}} \operatorname{Re}\left[\int_{0}^{\pi} d \theta \sin (\theta)\left[1+\epsilon_{d d}\left(3 \cos ^{2} \theta-1\right)\right]^{\frac{5}{2}}\right] .
$$

The eGPE Eq. (1) provides a reliable description of the available experimental phenomenology. In the absence of confinement in the transverse direction, this model predicts that for a certain value of the density and of $\epsilon_{d d}$, a phase transition between a uniform superfluid and a supersolid occurs. In the thermodynamic limit, the supersolid lattice is predicted to be triangular or honeycomb [40]. The occurrence of such a lattice symmetry has been also predicted for the case of transverse, radially symmetric, harmonic trapping $[32,43]$. Very recently, the possible existence of other exotic configurations in harmonic traps has been proposed $[44,45]$.

\section{CIRCULAR BOX POTENTIAL}

We first consider the case where the transverse confinement in the $x-y$ plane is provided by a circular box, while the confinement along the polarization direction ( $z$ axis) is of a harmonic nature. The case of a square box in the $x-y$ plane, as well as the properties of a quasi-one-dimensional box, will be discussed later. We always fix the height of the box potential to a value large enough to ensure that the density practically goes to zero at the border. Similar configurations have been already experimentally realized to trap alkali atoms [13-15]. In Fig. 1 we show examples of the ground-state density profiles [obtained by propagating Eq. (1) in imaginary time] of $N=10^{5}$ atoms of ${ }^{164} \mathrm{Dy}$ for different values of $\epsilon_{d d}$. As already anticipated, most of the atoms accumulate at the edge of the confining potential, forming a quasi-one-dimensional ring structure well separated from the atoms in the bulk. For small values of $\epsilon_{d d}$, both the edge and the bulk remain in the superfluid phase; while increasing $\epsilon_{d d}$ (i.e., increasing the effect of the dipolar force), the edge region clearly undergoes a phase transition to a supersolid phase, where the density peaks near the boundary of the box exhibit a finite overlap, ensuring global phase coherence. The overlap between the density peaks disappears for even larger values of $\epsilon_{d d}$, the system forming a sort of one-dimensional ring crystal.

The emergent edge ring geometry allows us to estimate the superfluid density in terms of the Leggett variational expression $[46,47]$. To this purpose we write the ground-state density in cylindrical coordinates $\rho(r, \theta, z)$, so that the Leggett's estimate for the superfluid density can be written as

$$
\frac{n_{S}}{n}=\frac{2 \pi}{n}\left(\int \frac{d \theta}{\int d r d z \rho(r, \theta, z)}\right)^{-1},
$$

where the integration over the radial coordinate is performed only in the edge region, identified by the density minima that appear both at the border of the box (where the density goes to zero) and at the interface between the edge and the bulk. In the case of the (extended) GPE, the estimate (4) for the superfluid density has been verified to coincide with the result obtained by imposing the twisted boundary condition to a one-dimensional array of droplets [48]. Remarkably, in Ref. [49] it has been shown that Leggett's estimate coincides with the exact superfluid density also in the case of peculiar stationary nonground-state solutions (cnoidal wave solution) which exhibit a periodic density modulation.

The estimate 4, reported in Fig. 2 (blue squares), reveals a critical dependence on $\epsilon_{d d}$, emphasizing the emergence of a phase transition between the superfluid and the supersolid phase at $\epsilon_{d d}=1.4$ and a transition between the supersolid and the crystal phase, characterized by the vanishing of $n_{S}$ [50] at $\epsilon_{d d}=1.55$. These values are very close to the critical values calculated for one-dimensional tubular configurations imposing periodic boundary conditions [24] after taking into account that in the edge configuration discussed here the number of atoms occupying the ring increases with $\epsilon_{d d}$, as shown in the same figure (red circles). While such a number is practically constant in the superfluid phase (although 


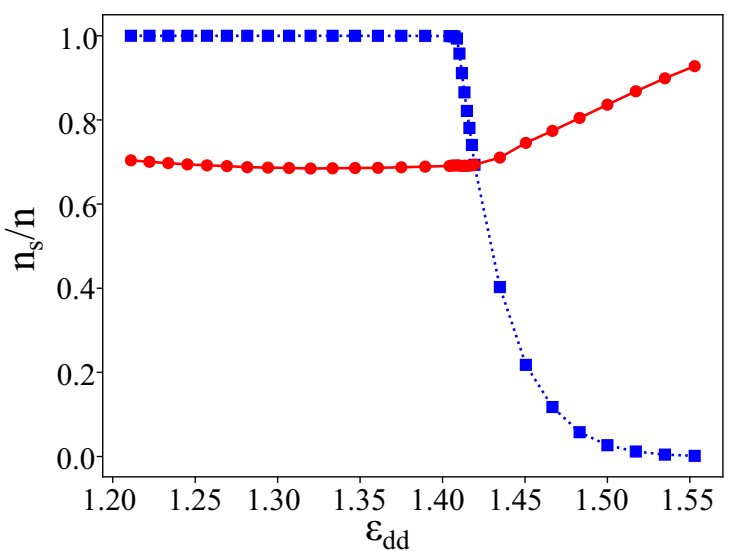

FIG. 2. Estimate of the superfluid fraction of the edge region as a function of $\epsilon_{d d}$, based on Leggett's variational formula 4 (blue squares), applied to the configuration described in Fig. 1. Red circles represent the ratio between the number of atoms that settle on the edge and the total number of atoms in the system

with a slight dependence on $\epsilon_{d d}$ as a consequence of small changes in the size of the edge), such an increase is actually particularly important in the supersolid phase as a consequence of the reduced value of the chemical potential, which favors the accumulation of dipoles on the density peaks, where the interatomic dipolar interaction is mainly attractive. We find here that the transition between the superfluid and the supersolid edge phase is continuous, as expected for a quasione-dimensional system, being characterized by the absence of a jump in the superfluid fraction at the critical value of $\epsilon_{d d}$.

The novel configuration emerging in the box of circular shapes discussed above is particularly attractive because in this case the boundary does not depend on the azimuthal coordinate and takes the form of a ring, where the dipolar particles form a one-dimensional structure, well separated from the atoms in the bulk. This provides the interesting possibility of exploring superfluid and supersolid features in uniform one-dimensional-like configurations with periodic boundary conditions, yielding an interesting alternative to the use of toroidal traps, where the nature of the superfluidsupersolid transition (continuous or discontinuous) depends crucially also on the transverse confinement [51]. Moreover, the presence of the low-density superfluid bulk paves the way to study interaction effects at the interface between a supersolid edge and a superfluid bulk. For instance, an interesting problem concerns the possibility of identifying stable modes, like sound waves or solitons, propagating only at the edge. Moreover, the superfluid-supersolid interface provides an ideal platform for the study of the so-called "crystallization waves," extensively studied at the liquid-solid interface in helium-4 [52]. In our case, due to the existence of a supersolid (not only a solid), one expects a very different behavior and possibly anomalous effects.

\section{SQUARE BOX POTENTIAL}

It is interesting to consider other forms of boxes like, for example, the most familiar square box. This case was considered in Ref. [39] in the deep superfluid phase and in the
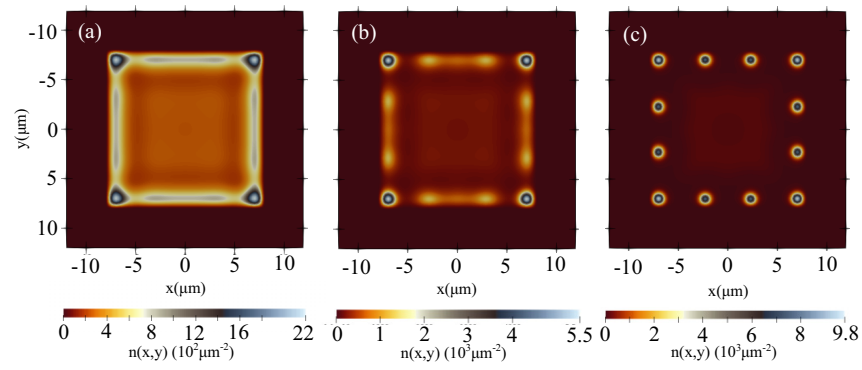

FIG. 3. Ground-state integrated density profiles $n(x, y)=$ $\int d z|\Psi(x, y, z)|^{2}$ for a gas of $10^{5}$ atoms of ${ }^{164}$ Dy confined in the polarization direction by a harmonic potential of frequency $\omega_{z}=(2 \pi) 100 \mathrm{~Hz}$ and by a box potential in the $\mathrm{x}-\mathrm{y}$ plane, with the shape of a square of side $L=16 \mu \mathrm{m}$. The value of $\epsilon_{d d}$ for (a)-(c) is, respectively, $1.32,1.404$, and 1.467 . The height of the box is fixed to $V_{0}=100 \hbar \omega_{z}$

absence of beyond-mean-field effects. Here we consider also regimes where the mean-field approach would yield instability and the LHY correction allows for the emergence of the supersolid and crystal phases.

The results for the density profiles in the case of a twodimensional square box are reported in Fig. 3 and reveal the same mechanism of accumulation of the density near the boundary already discussed in the case of a circular box. A major difference concerns the behavior of the density profile along the edge of the box. In fact the vertices of the square box become points of strong accumulation of dipoles, causing density modulations along the sides of the square, even for small values of $\epsilon_{d d}$ [see Fig. 3(a)] when the system is in the superfluid phase, thereby preventing the possibility of realizing uniform one dimensional superfluid configurations. The behavior of the density along each edge of the square configuration actually shares interesting analogies with the behavior exhibited by a quasi-one-dimensional gas confined by a box potential.

In order to obtain a deeper insight on this behavior, we compare the edge configurations in the case of the square box potential with a quasi-one-dimensional configuration confined by a box potential in the elongated direction and by a harmonic trap in the transverse direction. Typical density profiles for different values of $\epsilon_{d d}$ are reported in Figs. 4(a)-4(c), where we also report the density profiles calculated imposing, instead of a box potential, periodic boundary conditions at the edges of the simulation cell [panels (d)-(f)] as well as the corresponding excitation spectra [insets $(\mathrm{g})$ and $(\mathrm{h})$ ] calculated in the uniform phase by solving the Bogoliubov equations. The latter are obtained by linearizing the timedependent eGPE around the ground states (details on the procedure for dipolar gases described by the eGPE can be found in Refs. [24,53,54]). The configurations [panels (d) and (e)] correspond to a superfluid phase, the latter being characterized by a pronounced roton minimum, precursor of the instability to a periodically modulated density (supersolid phase) for larger values of $\epsilon_{d d}$ [panel (f)]. In the presence of the box, atoms accumulate close to the walls even for small values of $\epsilon_{d d}$ [weakly interacting dipolar case; panels (a), (d), 


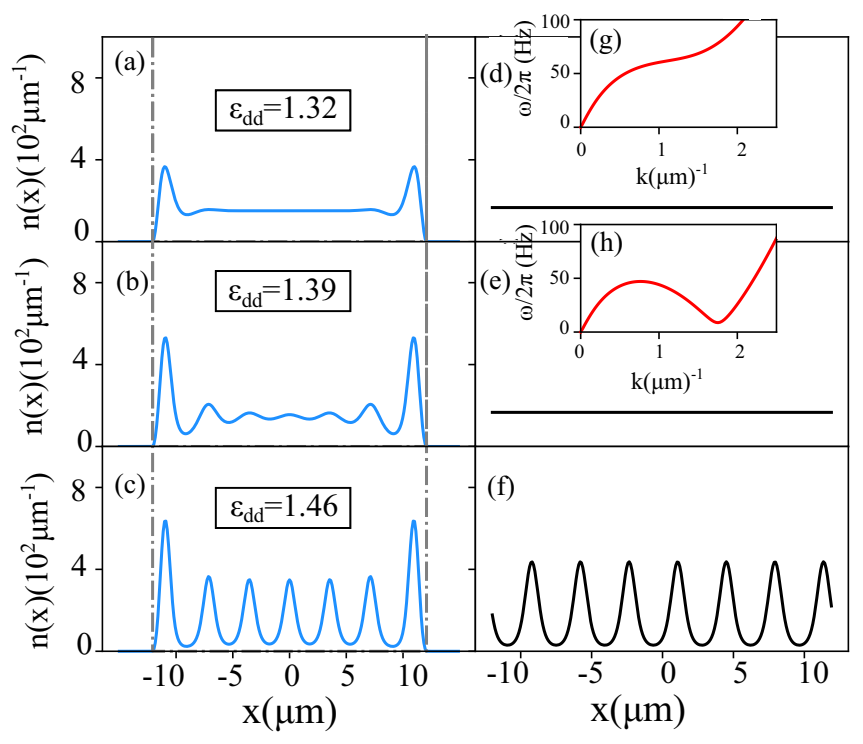

FIG. 4. Ground-state integrated density profiles $n(x)=$ $\int d y d z|\Psi(x, y, z)|^{2}$ of $\mathrm{N}=4 \times 10^{4}$ atoms of ${ }^{164} \mathrm{Dy}$ in a transverse harmonic confinement of frequencies $\omega_{y}=\omega_{z}=(2 \pi) 100 \mathrm{~Hz}$ confined by a one-dimensional box potential of height $V_{0}=100 \hbar \omega_{z}$ at positions $\mathrm{x}= \pm 12 \mu \mathrm{m}[(\mathrm{a})-(\mathrm{c})]$ or with periodic boundary conditions at $\mathrm{x}= \pm 12 \mu \mathrm{m}[(\mathrm{d})-(\mathrm{f})]$. Insets (g) and (h) show the excitation spectrum calculated by solving the Bogolyubov-de Gennes equations for the configurations of panels (d) and (e). The profiles reported in panels (a) and (d) [respectively, (b), (e), and (c), (f)] are calculated by fixing $\epsilon_{d d}=1.32$ (respectively, 1.39 and 1.46).

and inset $(\mathrm{g})$ ] when the excitation spectrum of the uniform phase does not show a roton minimum.

Due to the long range and anisotropic nature of the dipolar force, even in this case the density profile deeply differs from the results holding for a one-dimensional BEC interacting with a short-range potential. In the latter case the density profile, near a hard wall located at $x=0$, is fixed by the healing length $\xi=\sqrt{\hbar^{2} / 2 m g \bar{n}}$ according to $n(x)=\bar{n} \tanh ^{2}(x / \sqrt{2} \xi)$ where $\bar{n}$ is the bulk density away from the edge of the box [1]. The concept of healing length is not easily applicable to the case of a dipolar gas, whose different behavior is due to the long-range nature of the force, the presence, for large values of $\epsilon_{d d}$, of rotonic oscillations, and, of course, the emergence of spontaneous density modulations characterizing the supersolid and the crystal phases. The emergence of the rotonic oscillations is reminiscent of a similar effect characterizing the density profile in the vicinity of a quantized vortex [55]. This effect, originally theoretically investigated for quantized vortices in superfluid helium, is a direct consequence of the presence of the roton in the excitation spectrum [56-58]. The qualitatively similar behavior observed along each edge of the square box potential shown in Fig. 3 suggests that such edge configurations host, between two vertices of the confining potential, localized excitations corresponding to those that naturally occur in quasi-one-dimensional configurations.

The above discussion reveals that the presence of the square box makes the identification of the transition between the superfluid, the supersolid, and the crystal phases of the

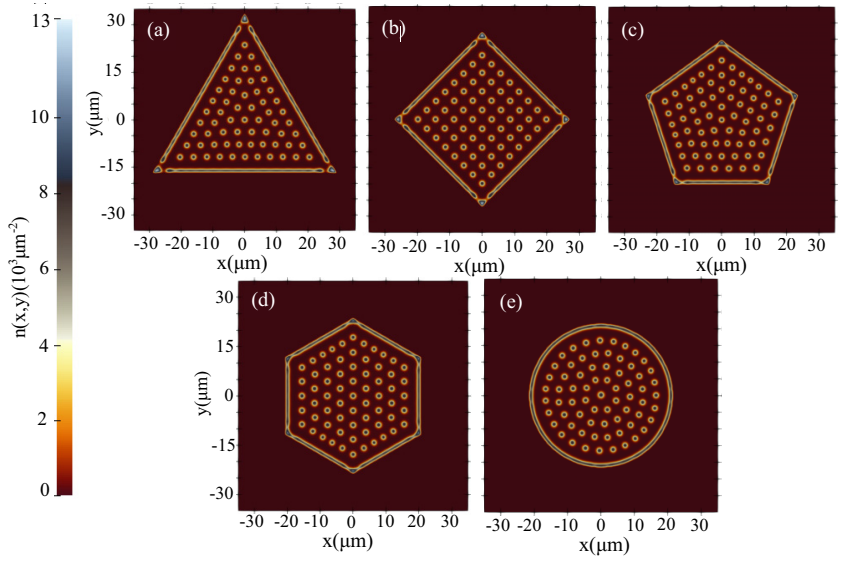

FIG. 5. Ground-state integrated density profiles $n(x, y)=$ $\int d z|\Psi(x, y, z)|^{2}$ for a gas of $2 \times 10^{6}$ atoms of ${ }^{164}$ Dy confined in the polarization direction by a harmonic potential of frequency $\omega_{z}=(2 \pi) 100 \mathrm{~Hz}$ and by a box potential in the $x-y$ plane with the shape of a triangle of side $L=58.58 \mu \mathrm{m}$ (a), a square of side $L=45.84 \mu \mathrm{m}$ (b), a pentagon of side $L=38.33 \mu \mathrm{m}$ (c), a hexagon of side $L=33.82 \mu \mathrm{m}(\mathrm{d})$, and a circle of radius $R=21.75 \mu \mathrm{m}$ (e). The value of $\epsilon_{d d}=1.36$ is the same for all the configurations, which also have the same area.

dipolar gas on the edge of the box more difficult than in the case of the circular box.

\section{BULK SUPERSOLIDITY}

The configurations discussed so far do not reveal the emergence of supersolid effects in the bulk region because of the small value of the bulk density caused by the accumulation of dipoles near the boundary. In order to observe bulk supersolidity, one consequently needs to increase significantly the atom density in such a way that the density in the central region remains large enough to ensure the appearance of a crystal quantum phase. In Fig. 5, we consider configurations containing $N=2 \times 10^{6}$ atoms confined by a box potential in the transverse direction, with the shape of regular polygons [panels (a)-(d)] or circular [panel (e)], all with the same area (and hence the same number of atoms per unit surface). For the same value of $\epsilon_{d d}=1.36$, these configurations exhibit a supersolid structure in the bulk, characterized by the typical overlap between neighboring density peaks, well separated from the edge region by a density dip. Despite the number of atoms and system size considered, resulting in a large number of droplets $(\simeq 60)$, the symmetry of the supersolid lattice reflects one of the confining potentials, implying that surface effects hinder the possibility of reaching the thermodynamic limit where the lattice is expected to be triangular or honeycomb [40]. This can be qualitatively understood as a consequence of the long-range nature of the dipolar force and the formation of the edge. In fact, since the dipoles are in a mainly repulsive configuration, they tend to expand toward the edge where they acquire a density profile with the same shape of the confining potential; the droplets that form in the bulk also tend to repel each other, but their expansion is stopped by the repulsion of the edge so that they are forced to arrange in lines parallel to the sides of the edge. This behavior 


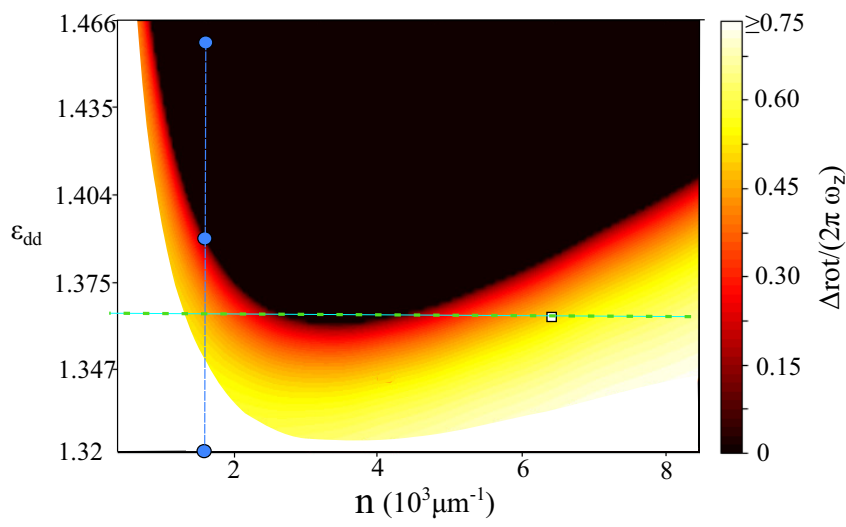

FIG. 6. Roton energy of a quasi-one-dimensional dipolar Bose gas with transverse harmonic confinement of frequencies $\omega_{y}=\omega_{z}=$ $(2 \pi) 100 \mathrm{~Hz}$, with periodic boundary conditions along the $x$ axis, as function of the linear density $n=N / L$, where $\mathrm{N}$ is the number of atoms and $\mathrm{L}$ is the length of the simulation cell along $x$ and of $\epsilon_{d d}$. The blue dots correspond, from bottom to top, to the configurations of Figs. 4(d)-4(f), while the white empty square corresponds to the ring edge of the configuration shown in Fig. 5(e) (circular transverse confinement). The dark area corresponds to configurations in which the roton mode, calculated starting from a uniform configuration, is unstable and where spontaneous density modulations of supersolid or crystal nature are formed. The green, horizontal dashed line is a guide to the eye showing that, for fixed $\epsilon_{d d}$, starting from a low-density superfluid, increasing the density results in a phase transition to a supersolid (and eventually to a droplet crystal), while at even higher densities, the system turns superfluid again.

is suppressed in a harmonic trap where the expansion of the gas is energetically unfavorable.

Notice that in our case the emergence of periodic density modulations in the bulk region is the result of the interatomic forces, which cause the spontaneous breaking of translational invariance. This implies, for example, that, different from the case of a lattice supersolid, the bulk supersolids with exotic lattice structures described above can propagate sound via lattice phonons.

It is worth noticing that the supersolid and crystal structures at the edge of the boundary, which are well visible in the configurations of Figs. 1 and 3(b) and 3(c), have disappeared in Fig. 5 as a consequence of the high density acquired by the system near the boundary, caused by the large value of $N$. This behavior reminds us once again the case of a quasi-one-dimensional configuration characterized by transverse harmonic confinement and periodic boundary conditions along the elongated direction. In this case, the value of the roton energy depends in a nonmonotonic way on both the linear density $n=N / L$, where $\mathrm{N}$ is the total number of atoms and $\mathrm{L}$ is the length of the simulation cell and $\epsilon_{d d}$ [51]. For a fixed external trapping in the $y-z$ plane, the stability region of the roton in fact acquires a characteristic, asymmetric, and inverse-bell shape, as shown in Fig. 6 (see also Ref. [51]). This implies that, for a fixed value of $\epsilon_{d d}$, increasing the density starting from small values, the system first enters the instability region of the roton mode [black region of Fig. 6(b)], to come back to the stable region, characterized again by the occurrence of a roton with a finite excitation energy, at larger values of the density. The coexistence of a supersolid bulk and a superfluid edge open another interesting possibility of exploring the physics of a superfluid-supersolid interface. This effect can also be observed with a smaller number of atoms by confining the atoms in properly designed box potentials of smaller dimension. In fact, such density, although relatively high $\left(\simeq 10^{15} \mathrm{~cm}^{-3}\right.$ for the edge configurations shown in Fig. 5), is still compatible with the usual stability conditions imposed by three-body recombination, suggesting the possibility of observing this effect in actual experiments.

We have finally checked that the results presented in this work do not qualitatively change for different choices of the parameters. In particular we have considered different values of the confinement frequency in the polarization direction in the interval $(2 \pi) 50 \mathrm{~Hz}<\omega_{z}<(2 \pi) 150 \mathrm{~Hz}$ and of system size and number of atoms. The actual choice of $\omega_{z}$ can, however, affect the value of the density in the central region, the critical value of $\epsilon_{d d}$ for the superfluid-supersolid phase transition, as well as the number of droplets which form in the supersolid phase, their relative distance being sensitive to the value of $\omega_{z}$ [23].

Finally, we have checked that by properly tuning the interaction parameters and number of atoms, it is possible to obtain also other bulk-edge interfaces beside the more interesting hybrid superfluid-supersolid one. For example, for a fixed number of atoms, in the configurations of Fig. 5, reducing $\epsilon_{d d}$ below a certain critical value results in a superfluid-superfluid interface, while increasing it above another critical value results in a configuration where both the bulk and the edge spontaneously develop a periodic density modulation.

In conclusion, we have investigated the ground-state configurations of a dipolar Bose-Einstein condensed gas confined by a box potential. We have shown that the tendency of the density to accumulate near the walls, as a consequence of the repulsion between aligned dipoles, favors the formation of novel quasi-one-dimensional configurations located at the edge of the box and well separated from the atoms filling the bulk region. In the case of quasi-two-dimensional boxes of circular shape, the edge configuration takes the characteristic form of a ring, revealing clear supersolid and crystal effects in a useful range of parameters. We have also shown that the geometry of the supersolid in the bulk region reflects the shape of the confining potential even for very large systems, therefore hindering the possibility of reaching the thermodynamic limit of dipolar BECs using box potentials. A natural extension of this work concerns the study of the nonequilibrium behavior exhibited by dipolar gases in the novel ring configuration formed at the edge of the circular box.

\section{ACKNOWLEDGMENTS}

Useful discussions with G. Modugno and the members of the Firenze-Pisa dipolar group are acknowledged. This project received funding from Provincia Autonoma di Trento, the $\mathrm{Q} @ \mathrm{TN}$ initiative and the FIS $\hbar$ project of the Istituto Nazionale di Fisica Nucleare, and the Italian MIUR under the PRIN2017 Project CEnTraL. 
[1] L. P. Pitaevskii and S. Stringari, Bose-Einstein Condensation and Superfluidity (Oxford University Press, Oxford, 2016).

[2] S. Stringari, Collective Excitations of a Trapped BoseCondensed Gas, Phys. Rev. Lett. 77, 2360 (1996).

[3] M.-O. Mewes, M. R. Andrews, N. J. van Druten, D. M. Kurn, D. S. Durfee, C. G. Townsend, and W. Ketterle, Collective Excitations of a Bose-Einstein Condensate in a Magnetic Trap, Phys. Rev. Lett. 77, 988 (1996).

[4] D. Guéry-Odelin and S. Stringari, Scissors Mode and Superfluidity of a Trapped Bose-Einstein Condensed Gas, Phys. Rev. Lett. 83, 4452 (1999).

[5] O. M. Maragò, S. A. Hopkins, J. Arlt, E. Hodby, G. Hechenblaikner, and C. J. Foot, Observation of the Scissors Mode and Evidence for Superfluidity of a Trapped BoseEinstein Condensed Gas, Phys. Rev. Lett. 84, 2056 (2000).

[6] C. D. Rossi, R. Dubessy, K. Merloti, M. de Goër de Herve, T. Badr, A. Perrin, L. Longchambon, and H. Perrin, Probing superfluidity in a quasi two-dimensional Bose gas through its local dynamics, New J. Phys. 18, 062001 (2016).

[7] K. W. Madison, F. Chevy, W. Wohlleben, and J. Dalibard, Vortex Formation in a Stirred Bose-Einstein Condensate, Phys. Rev. Lett. 84, 806 (2000).

[8] J. R. Abo-Shaeer, C. Raman, J. M. Vogels, and W. Ketterle, Observation of vortex lattices in Bose-Einstein condensates, Science 292, 476 (2001)

[9] P. C. Haljan, I. Coddington, P. Engels, and E. A. Cornell, Driving Bose-Einstein-Condensate Vorticity with a Rotating Normal Cloud, Phys. Rev. Lett. 87, 210403 (2001).

[10] T. P. Meyrath, F. Schreck, J. L. Hanssen, C.-S. Chuu, and M. G. Raizen, Bose-Einstein condensate in a box, Phys. Rev. A 71, 041604(R) (2005).

[11] A. L. Gaunt, T. F. Schmidutz, I. Gotlibovych, R. P. Smith, and Z. Hadzibabic, Bose-Einstein Condensation of Atoms in a Uniform Potential, Phys. Rev. Lett. 110, 200406 (2013).

[12] S. Gupta, K. W. Murch, K. L. Moore, T. P. Purdy, and D. M. Stamper-Kurn, Bose-Einstein Condensation in a Circular Waveguide, Phys. Rev. Lett. 95, 143201 (2005).

[13] N. Navon, A. L. Gaunt, R. P. Smith, and Z. Hadzibabic, Critical dynamics of spontaneous symmetry breaking in a homogeneous Bose gas, Science 347, 167 (2015).

[14] L. Chomaz, L. Corman, T. Bienaimé, R. Desbuquois, C. Weitenberg, S. Nascimbène, J. Beugnon, and J. Dalibard, Emergence of coherence via transverse condensation in a uniform quasi-two-dimensional Bose gas, Nat. Commun. 6, 6162 (2015).

[15] N. Navon, A. L. Gaunt, R. P. Smith, and Z. Hadzibabic, Emergence of a turbulent cascade in a quantum gas, Nature (London) 539, 72 (2016).

[16] J. L. Ville, T. Bienaimé, R. Saint-Jalm, L. Corman, M. Aidelsburger, L. Chomaz, K. Kleinlein, D. Perconte, S. Nascimbène, J. Dalibard, and J. Beugnon, Loading and compression of a single two-dimensional bose gas in an optical accordion, Phys. Rev. A 95, 013632 (2017).

[17] J. L. Ville, R. Saint-Jalm, E. Le Cerf, M. Aidelsburger, S. Nascimbène, J. Dalibard, and J. Beugnon, Sound Propagation in a Uniform Superfluid Two-Dimensional Bose Gas, Phys. Rev. Lett. 121, 145301 (2018).

[18] A. Griesmaier, J. Werner, S. Hensler, J. Stuhler, and T. Pfau, Bose-Einstein Condensation of Chromium, Phys. Rev. Lett. 94, 160401 (2005).
[19] E. Lucioni, L. Tanzi, A. Fregosi, J. Catani, S. Gozzini, M. Inguscio, A. Fioretti, C. Gabbanini, and G. Modugno, Dysprosium dipolar Bose-Einstein condensate with broad feshbach resonances, Phys. Rev. A 97, 060701(R) (2018).

[20] M. Lu, N. Q. Burdick, S. H. Youn, and B. L. Lev, Strongly Dipolar Bose-Einstein Condensate of Dysprosium, Phys. Rev. Lett. 107, 190401 (2011).

[21] K. Aikawa, A. Frisch, M. Mark, S. Baier, A. Rietzler, R. Grimm, and F. Ferlaino, Bose-Einstein Condensation of Erbium, Phys. Rev. Lett. 108, 210401 (2012).

[22] T. Koch, T. Lahaye, J. Metz, B. Fröhlich, A. Griesmaier, and T. Pfau, Stabilization of a purely dipolar quantum gas against collapse, Nat. Phys. 4, 218 (2008).

[23] L. Santos, G. V. Shlyapnikov, and M. Lewenstein, Roton-Maxon Spectrum and Stability of Trapped Dipolar Bose-Einstein Condensates, Phys. Rev. Lett. 90, 250403 (2003).

[24] S. M. Roccuzzo and F. Ancilotto, Supersolid behavior of a dipolar Bose-Einstein condensate confined in a tube, Phys. Rev. A 99, 041601(R) (2019).

[25] L. Chomaz, R. M. W. van Bijnen, D. Petter, G. Faraoni, S. Baier, J. H. Becher, M. J. Mark, F. Wächtler, L. Santos, and F. Ferlaino, Observation of roton mode population in a dipolar quantum gas, Nat. Phys. 14, 442 (2018).

[26] D. Petter, G. Natale, R. M. W. van Bijnen, A. Patscheider, M. J. Mark, L. Chomaz, and F. Ferlaino, Probing the Roton Excitation Spectrum of a Stable Dipolar Bose Gas, Phys. Rev. Lett. 122, 183401 (2019).

[27] I. Ferrier-Barbut, H. Kadau, M. Schmitt, M. Wenzel, and T. Pfau, Observation of Quantum Droplets in a Strongly Dipolar Bose Gas, Phys. Rev. Lett. 116, 215301 (2016).

[28] H. Kadau, M. Schmitt, M. Wenzel, C. Wink, T. Maier, I. Ferrier-Barbut, and T. Pfau, Observing the rosensweig instability of a quantum ferrofluid, Nature (London) 530, 194 (2016).

[29] I. Ferrier-Barbut, M. Schmitt, M. Wenzel, H. Kadau, and T. Pfau, Liquid quantum droplets of ultracold magnetic atoms, J. Phys. B: At. Mol. Opt. Phys. 49, 214004 (2016).

[30] M. Schmitt, M. Wenzel, F. Böttcher, I. Ferrier-Barbut, and T. Pfau, Self-bound droplets of a dilute magnetic quantum liquid, Nature (London) 539, 259 (2016).

[31] L. Tanzi, E. Lucioni, F. Famà, J. Catani, A. Fioretti, C. Gabbanini, R. N. Bisset, L. Santos, and G. Modugno, Observation of a Dipolar Quantum Gas with Metastable Supersolid Properties, Phys. Rev. Lett. 122, 130405 (2019).

[32] S. M. Roccuzzo, A. Gallemí, A. Recati, and S. Stringari, Rotating a Supersolid Dipolar Gas, Phys. Rev. Lett. 124, 045702 (2020).

[33] L. Chomaz, D. Petter, P. Ilzhöfer, G. Natale, A. Trautmann, C. Politi, G. Durastante, R. M. W. van Bijnen, A. Patscheider, M. Sohmen, M. J. Mark, and F. Ferlaino, Long-Lived and Transient Supersolid Behaviors in Dipolar Quantum Gases, Phys. Rev. X 9, 021012 (2019).

[34] G. Natale, R. M. W. van Bijnen, A. Patscheider, D. Petter, M. J. Mark, L. Chomaz, and F. Ferlaino, Excitation Spectrum of a Trapped Dipolar Supersolid and its Experimental Evidence, Phys. Rev. Lett. 123, 050402 (2019).

[35] F. Böttcher, J.-N. Schmidt, M. Wenzel, J. Hertkorn, M. Guo, T. Langen, and T. Pfau, Transient Supersolid Properties in an 
Array of Dipolar Quantum Droplets, Phys. Rev. X 9, 011051 (2019).

[36] M. Guo, F. Böttcher, J. Hertkorn, J.-N. Schmidt, M. Wenzel, H. P. Büchler, T. Langen, and T. Pfau, The low-energy Goldstone mode in a trapped dipolar supersolid, Nature (London) 574, 386 (2019).

[37] J. Léonard, A. Morales, P. Zupancic, T. Esslinger, and T. Donner, Supersolid formation in a quantum gas breaking a continuous translational symmetry, Nature (London) 543, 87 (2017).

[38] J.-R. Li, J. Lee, W. Huang, S. Burchesky, B. Shteynas, F. Ç. Top, A. O. Jamison, and W. Ketterle, A stripe phase with supersolid properties in spin-orbit-coupled Bose-Einstein condensates, Nature (London) 543, 91 (2017).

[39] H.-Y. Lu, H. Lu, J.-N. Zhang, R.-Z. Qiu, H. Pu, and S. Yi, Spatial density oscillations in trapped dipolar condensates, Phys. Rev. A 82, 023622 (2010).

[40] Y.-C. Zhang, F. Maucher, and T. Pohl, Supersolidity Around a Critical Point in Dipolar Bose-Einstein Condensates, Phys. Rev. Lett. 123, 015301 (2019).

[41] A. R. P. Lima and A. Pelster, Beyond mean-field low-lying excitations of dipolar Bose gases, Phys. Rev. A 86, 063609 (2012).

[42] F. Wächtler and L. Santos, Quantum filaments in dipolar Bose-Einstein condensates, Phys. Rev. A 93, 061603(R) (2016).

[43] A. Gallemí, S. M. Roccuzzo, S. Stringari, and A. Recati, Quantized vortices in dipolar supersolid Bose-Einstein-condensed gases, Phys. Rev. A 102, 023322 (2020).

[44] J. Hertkorn, J. N. Schmidt, M. Guo, F. Böttcher, K. S. H. Ng, S. D. Graham, P. Uerlings, T. Langen, M. Zwierlein, and T. Pfau, Pattern formation in quantum ferrofluids: from supersolids to superglasses, Phys. Rev. Research 3, 033125 (2021).
[45] Y.-C. Zhang, T. Pohl, and F. Maucher, Phases of supersolids in confined dipolar Bose-Einstein condensates, Phys. Rev. A 104, 013310 (2021).

[46] A. J. Leggett, Can a Solid be "Superfluid"? Phys. Rev. Lett. 25, 1543 (1970).

[47] A. J. Leggett, On the superfluid fraction of an arbitrary manybody system at T = 0, J. Stat. Phys. 93, 927 (1998).

[48] S. Roccuzzo, Supersolidity in a dipolar Bose gas, Ph.D. thesis, University of Trento, 2021.

[49] G. I. Martone, A. Recati, and N. Pavloff, Supersolidity of cnoidal waves in an ultracold bose gas, Phys. Rev. Research 3, 013143 (2021).

[50] Here, we consider an arbitrarily chosen small value of $n_{S} / n \leqslant$ $10^{-2}$.

[51] P. B. Blakie, D. Baillie, L. Chomaz, and F. Ferlaino, Supersolidity in an elongated dipolar condensate, Phys. Rev. Research 2, 043318 (2020).

[52] S. Balibar, H. Alles, and A. Y. Parshin, The surface of helium crystals, Rev. Mod. Phys. 77, 317 (2005).

[53] F. Ancilotto, M. Rossi, and F. Toigo, Supersolid structure and excitation spectrum of soft-core bosons in three dimensions, Phys. Rev. A 88, 033618 (2013).

[54] D. Baillie, R. M. Wilson, and P. B. Blakie, Collective Excitations of Self-Bound Droplets of a Dipolar Quantum Fluid, Phys. Rev. Lett. 119, 255302 (2017).

[55] M. Abad, M. Guilleumas, R. Mayol, M. Pi, and D. M. Jezek, Vortices in Bose-Einstein condensates with dominant dipolar interactions, Phys. Rev. A 79, 063622 (2009).

[56] T. Regge, Free boundary of he ii and Feynman wave functions, J. Low Temp. Phys. 9, 123 (1972).

[57] F. Dalfovo, Structure of vortices in helium at zero temperature, Phys. Rev. B 46, 5482 (1992).

[58] G. V. Chester, R. Metz, and L. Reatto, On the theory of quantized vortices, Phys. Rev. 175, 275 (1968). 